\title{
A METHOD OF DETERMINING THE DEW POINTS OF FUEL-AIR MIXTURES
}

\author{
By Roy J. Kennedy
}

\section{ABSTRACT}

A limit to the proportions of low-volatility constituents permissible in a hydrocarbon fuel is imposed by the temperature necessary to keep a mixture of the fuel and air completely vaporized. This paper is an outline of a method developed at the Bureau of Standards for determining the minimum temperatures necessary for the purpose.

The theory of the method rests on the assumption that the initial condensate in equilibrium with the remaining vapor is of essentially constant composition for the range of pressures and temperatures encountered in the engine manifold. The Clausius-Clapeyron equation and the ideal gas laws are then applied and an expression is developed for the temperature sought in terms of the pressure and mixture ratio of the fuel-air mixture. The expression involves several constants characteristic of the fuel, which are determined by experimental methods described in the paper.

\section{CONTENTS}

I. Introduction

II. Theory of method. 49

III. Apparatus and method......... 53

IV. Comparison of methods.

\section{INTRODUCTION}

Problems arising in the design of internal-combustion engines and in the engine laboratory have necessitated the determination of the minimum temperatures required to prevent condensation from explosive mixtures of air with the various hydrocarbon fuels. These temperatures are called the dew points of the mixtures. They are the same as the temperatures necessary to just effect the vaporization of the last particles of liquid fuel in the presence of the vapor mixture containing the rest of the fuel. Of course, the dew point is quite distinct from the dry point or the end point obtained in an ordinary distillation where the vapor does not remain in contact with the liquid phase. In that case the composition of the last of the liquid is very different from that we are here concerned with, if the fuel is a solution of several components. 
The dew points can be computed from the vapor pressure of the fuel when it consists of a single compound. When it is a solution, the condensation temperatures can not, in general, be inferred from its vapor pressure or the vapor pressures of its components, even if their proportions in the solution are known. The composition of a gasoline is usually not even qualitatively known.

Wilson and Barnard ${ }^{1}$ have devised an ingenious indirect method and W. A. Gruse ${ }^{2}$ a direct method of determining the dew points

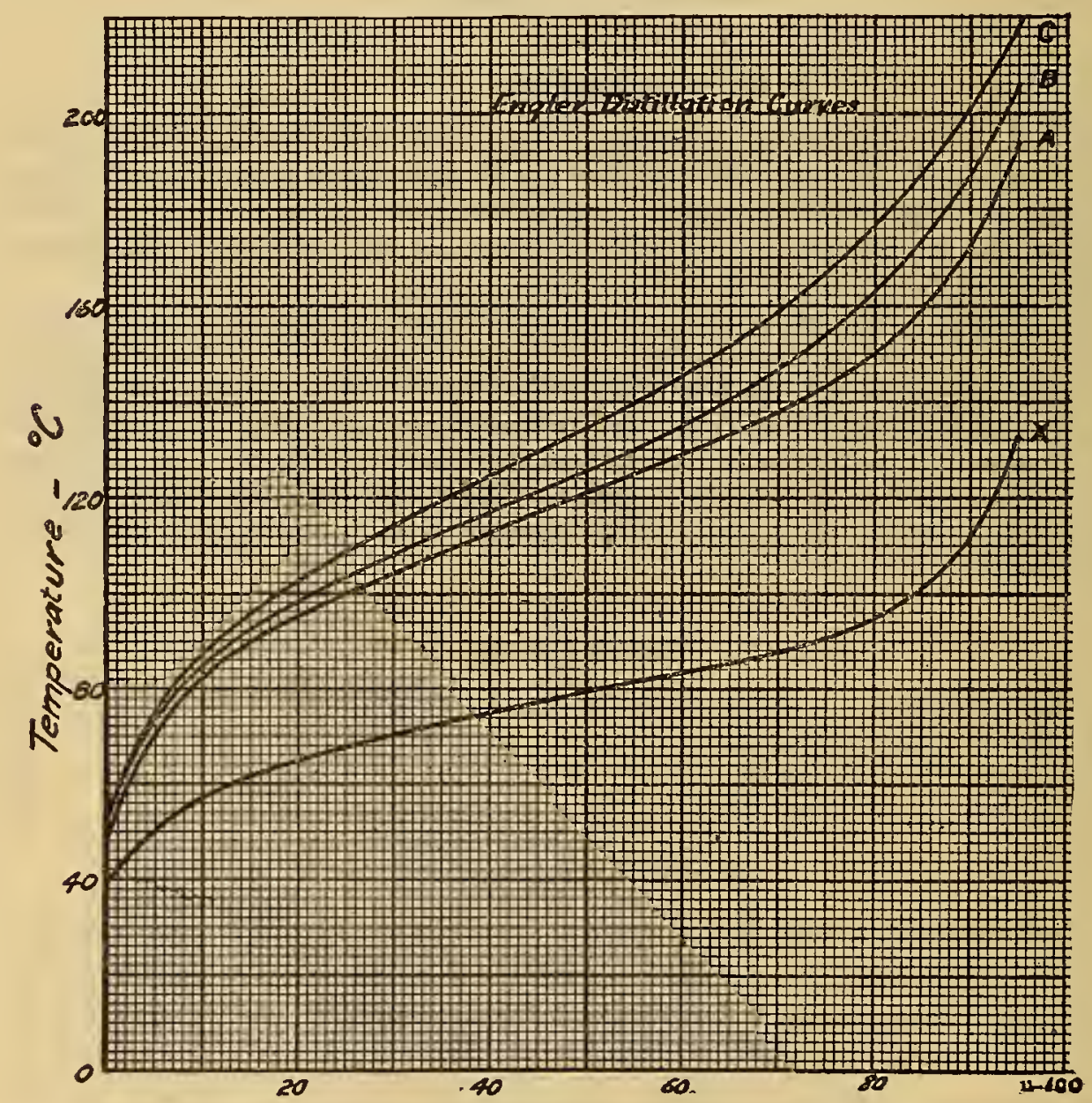

FIG. 1.-Distillation curves of Bureau of Standards fuels

in terms of the mixture ratio and the pressure exerted by the mixture.

In Gruse's method the completely vaporized fuel-air mixture, formed in the desired proportions by vaporizing the fuel at a known rate and mixing it with a current of heated air at the desired pressure, is passed over a polished surface whose temperature is adjusted till dew just appears or disappears. This temperature is the dew point for the fuel under the given conditions. In Wilson and Barnard's method there is derived from the fuel by distillation a so-called

1 Contribution No. 36 from Research Laboratory of A pplied Chemistry, Mass. Inst. of Tech.

' Industrial and Engineering Chemistry, p. 796, August, 1923. 
equilibrium solution which is intended to represent the first portion of the mixture condensing at the dew point. The vapor pressure of this solution is measured over a convenient temperature range and assumed to equal the pressure exerted by an amount of fuel which would be just completely vaporized at corresponding temperatures; that is, the partial pressure of the fuel at corresponding dew points of the mixture. From the partial pressure and the average molecular weight of the fuel (determined approximately from distillation data) the dew point of the mixture can be computed as a function of the mixture ratio and total pressure.

The method forming the subject of this paper was devised by W. S. James, of this bureau, and the writer. To test it for consistency, and to check the results obtained by other methods, dewpoint determinations have been made on absolute alcohol, two gasolines, P. and G. G., supplied through the courtesy of Doctor Gruse, of the Mellon Institute of Industrial Research of the University of Pittsburgh, an aviation gasoline (marked " $\mathrm{X}$-gasoline" in the curves) and the automobile fuels (marked $A, B$, and $C$ ) used in an extended investigation of economic volatility at the bureau. Curves are included for only the last four (fig. 1), the others being reported elsewehere. ${ }^{3}$ The primary object has been to test the method rather than to accumulate data.

\section{THEORY OF METHOD}

The theory of the method rests on the assumption that over the range of pressures here dealt with (those occurring in the engine manifold) the composition of the first observable portion of liquid condensing just below the dew point remains sensibly constant; that is, that throughout this range the initial condensate is the same substance. This fraction being a true equilibrium solution, the proportions of its constituents must, in general, vary to some extent, but, as will be shown hereafter, the variation appears to be negligible for this small range. It is therefore possible to apply to the condensate the Clausius-Clapeyron relation between its vapor pressure and temperature

where

$$
\frac{d p}{d T}=\frac{L}{\left(v-v^{\prime}\right) T}
$$

$p=$ vapor pressure exerted by initial condensate at absolute temperature $T$,

$L=$ molecular heat of vaporization of initial condensate at temperature at which vaporization occurs,

$v^{\prime}=$ molecular volume of initial condensate as liquid,

$v=$ molecular volume of initial condensate as vapor.

Sruse, loc. cit. 
The volume of a given mass of liquid is negligible as compared with the volume of same mass of vapor, so that $v^{\prime}$ may be dropped from the equation. Assuming the vapor to follow the laws of ideal gases (its pressure being a small fraction of an atmosphere), its molecular volume may be expressed as $v=\frac{R T}{p}$. Substituting this value in the equation we get

$$
\frac{d p}{d T}=\frac{L p}{R T^{2}}
$$

where $R$ is the universal gas constant. It is to be borne in mind that only when $T$ is the initial condensation temperature of the mixture do this relation and those deduced from it apply to the condensate. At other temperatures the vapor is either superheated or else of different composition than the original liquid, because not all of its constituents condense in the same proportions. Hence, the temperature occurring in the equations is the dew point.

Another equation, relating $L$ and the molecular heat at constant pressure, $C_{\mathrm{p}}$, of the vapor of the initial condensate, and the molecular heat, $C$, of the same substance in the liquid state is

$$
\frac{d L}{d T}=C_{\mathbf{p}}-C
$$

Both expressions are elementary thermodynamic deductions applying to all substances. They will be found derived in any textbook.

The molecular heats $C_{\mathrm{p}}$ and $C$ are, in general, unknown functions of the temperature, but for small temperature ranges they are nearly constant. Hence, their difference is here assumed constant and equation (2) becomes integrable. Its integral is

$$
L=\left(C_{\mathrm{p}}-C\right) T+k
$$

where $k$ is a constant. From (1)

$$
\begin{gathered}
L=\frac{R T^{2}}{p} \frac{d p}{d T} \\
\therefore \frac{1}{p} \frac{d p}{d T}=\frac{C_{\mathrm{p}}-C}{R T}+\frac{k}{R T^{2}}
\end{gathered}
$$

Integrating and rearranging

$$
\log p=\frac{k_{1}^{\prime}}{T}+\frac{C_{p}-C}{R} \log T+k^{\prime}
$$

where $k_{1}^{\prime}$ and $k_{2}^{\prime}$ are new constants. 
This expression can be simplified by the introduction of an approximation. In the neighborhood of any temperature $T_{\mathrm{o}}$, the relation

$$
\log T=1+\log T_{\mathrm{o}}-\frac{T_{\mathrm{o}}}{T}
$$

holds approximately. The error in $\log T$ thus involved is, for $T=T_{\mathrm{o}}+d T$

$$
\log T-\left(1+\log T_{\mathrm{o}}-\frac{T_{\mathrm{o}}}{T}\right)
$$

$$
\begin{aligned}
& =\log \frac{T}{T_{\mathrm{o}}}-1+\frac{T_{\mathrm{o}}}{T} \\
& =\log \frac{T_{\mathrm{o}}+d T}{T_{\mathrm{o}}}-1+\frac{T_{\mathrm{o}}}{T_{\mathrm{o}}+d T} \\
& =\log \left(1+\frac{d T}{T_{\mathrm{o}}}\right)-1+\frac{1}{1+\frac{d T}{T_{\mathrm{o}}}} \\
& =\frac{d T}{T_{\mathrm{o}}}-\frac{1}{2}\left(\frac{d T}{T_{\mathrm{o}}}\right)^{2}+\frac{1}{3}\left(\frac{d T}{T_{\mathrm{o}}}\right)^{3} \cdots-1+1-\frac{d T}{T_{\mathrm{o}}}+\left(\frac{d T}{T_{\mathrm{o}}}\right)^{2}-\left(\frac{d T}{T_{\mathrm{o}}}\right)^{3}+\cdots \\
& =\frac{1}{2}\left(\frac{d T}{T_{\mathrm{o}}}\right)^{2}-\frac{2}{3}\left(\frac{d T}{T_{\mathrm{o}}}\right)^{3}+\cdots
\end{aligned}
$$

This series rapidly converges to a value less than $\frac{1}{2}\left(\frac{d T}{T_{\mathrm{o}}}\right)^{2}$. The maximum value of this quantity for the temperatures in the present work is less than one-fourth of 1 per cent, so that the approximation is justified.

When the substitution from equation (4) is made for $\log T$ in equation (3), the latter becomes

$$
\log p=\frac{k^{\prime}{ }_{1}}{T}-\frac{C_{\mathrm{p}}-C}{R} \frac{T_{\mathrm{0}}}{T}+\frac{C_{\mathrm{p}}-C}{R}\left(1+\log T_{\mathrm{o}}\right)+k^{\prime}{ }_{2}=\frac{K_{1}}{T}+k_{2}
$$

Now at the dew point, the vapor pressure of the substance first condensing equals its partial pressure in the completely vaporized mixture. According to Dalton's law and the equation of condition $(p v=n R T)$ this latter pressure and the pressure exerted by the fuel as a whole are each proportional to the absolute temperature and consequently proportional to one another; that is,

$$
p=k_{3} P_{\mathrm{f}}
$$

if partial pressure of fuel as a whole is designated by $P_{\mathrm{f}}$ and $k_{3}$ is a constant. Substituting for $p$ in equation (5)

$$
\log P_{\mathrm{P}}=\frac{K_{1}}{T}+K_{2}
$$


The correctness of the assumption of constancy of composition of the initial condensate is confirmed when experimentally determined values of $\log P_{f}$ (or for convenience the logarithms of a constant times $P_{\mathrm{f}}$ ) are plotted against the reciprocals of the corresponding temperatures, forming graphs which are very nearly straight lines. Departures from linearity in the relation are within the limits of experimental error.

According to Dalton's law the total pressure of the fuel-air mixture is the sum of the partial pressures, $P_{\mathrm{f}}$ and $P_{\mathrm{a}}$, of the fuel vapor and air, respectively; that is,

$$
P=P_{\mathrm{f}}+P_{\mathrm{a}}=P_{\mathrm{f}}\left(1+\frac{P_{\mathrm{a}}}{P_{\mathrm{f}}}\right)
$$

Similarly, the partial pressures of fuel and air are themselves the sums of the partial pressures of their constituents; that is,

and

$$
P_{1}=\frac{m_{1}}{M_{1}} \frac{R T}{V}+\frac{m_{2}}{M_{2}} \frac{R T}{V}+\cdots+\frac{m_{\mathrm{n}}}{M_{\mathrm{n}}} \frac{R T}{V}=\frac{R T}{V} \sum_{\text {fuel }} \frac{m}{M}
$$

$$
P_{\mathrm{a}}=\frac{R T}{V} \sum_{\text {air }} \frac{m}{M}
$$

In these expressions $m$ and $M$ refer to the masses and molecular weights, respectively, of the various species of molecules of fuel and air, $V$ is the volume of the containing vessel, and the summations extend over all species present. Defining the average molecular weights $M_{P}$ and $M_{\mathbf{a}}$ by the relations

and

$$
\frac{m_{\mathrm{f}}}{M_{\mathrm{f}}}=\sum_{\text {fuel }} \frac{m}{\bar{M}}
$$

$$
\frac{m_{\mathrm{a}}}{M_{\mathrm{a}}}=\sum_{\text {air }} \frac{m}{\bar{M}}
$$

where $m_{1}$ and $m_{\mathrm{a}}$ are the respective masses of fuel and air in a given volume of mixture, we get on substituting in equation (8)

and

$$
P_{\mathrm{f}}=\frac{R T}{V} \frac{m_{\mathrm{f}}}{M_{\mathrm{f}}}
$$

$$
P_{\mathrm{a}}=\frac{R T}{V} \frac{m_{\mathrm{a}}}{M_{\mathrm{a}}}
$$

Substituting these values for $P_{\mathrm{f}}$ and $P_{\mathrm{a}}$ in equation (7)

$$
P=P_{\mathrm{f}}\left(1+\frac{m_{\mathrm{a}}}{m_{\mathrm{f}}} \cdot \frac{M_{\mathrm{f}}}{M_{\mathrm{a}}}\right)
$$


The ratio $\frac{m_{\mathrm{a}}}{m_{\mathrm{f}}}$ is commonly called the mixture ratio. Designating it by $R^{\prime}$, we have

$$
P=P_{\mathrm{f}}\left(1+\frac{M_{\mathrm{f}}}{M_{\mathrm{a}}} R^{\prime}\right)
$$

Then $\log P=\log P_{\mathrm{f}}+\log \left(1+\frac{M_{\mathrm{f}}}{M_{\mathrm{a}}} R^{\prime}\right)$ and on replacing $\log P_{\mathrm{f}}$ by its value from equation (6)

Or, finally

$$
\log P=\frac{K_{1}}{T}+\log \left(1+\frac{M_{\mathrm{P}}}{M_{\mathrm{a}}} \mathrm{R}^{\prime}\right)+\Pi_{2}
$$

$$
T=\frac{K_{1}}{\log P-\log \left(1+\frac{M_{\mathrm{f}}}{M_{\mathrm{a}}} R^{\prime}\right)-K_{2}}
$$

This equation expresses the dew point as a function of the mixture ratio and total pressure of the mixture when the constants $K_{1}, K_{2}$, and $M_{\mathrm{f}}$, which are characteristic of the individual fuels, have been determined. For the average molecular weight of air, $M_{a}$, the value 29 is taken from tables. The experimental determination of the constants is made with the apparatus and by the method which are described below.

\section{APPARATUS AND METHOD}

The apparatus is diagrammed in Figure 2. It consists essentially of a thin-walled glass vapor chamber of about 1,500 cc volume, to which is sealed a metal cover provided with a manually operated oscillating fan having thin, blackened vanes, a burette graduated to cubic millimeters, and an outlet for connection to a vacuum pump. The chamber is immersed in a water bath arranged to be heated by a gas flame and cooled by the addition of tap water, which is circulated through a few turns of a spiral of copper tubing placed in the bath, then brought into contact with a small circular area of the bottom of the chamber, and finally stirred in with the bath water, of which a part is displaced through an overflow outlet. By varying the rate of influx of water it is thus possible to cool the chamber while maintaining the small area cooler by any desired amount. In this way condensation can be made to occur over the cooler spot, the rest of the wall surface remaining dry (because of the decreased partial pressure of fuel left in vapor state), while the bath is brought a few degrees below the condensation temperature. Windows in opposite sides of the metal outer vessel permit illuminating and viewing the cool spot, the bottom of chamber being blackened on the outside to eliminate stray light.

$24300^{\circ}-25 \dagger-2$ 
One way of determining dew points with this apparatus would be to form mixtures of air and fuel in the vapor chamber in a series of mixture ratios and at various pressures, and to observe directly the minimum temperatures for no condensation. Such procedure would require a large number of observations. It has been employed only for checking results secured by the method outlined below.

An accurately measured volume of liquid fuel is admitted and allowed sufficient time to vaporize completely in the partially evacu-

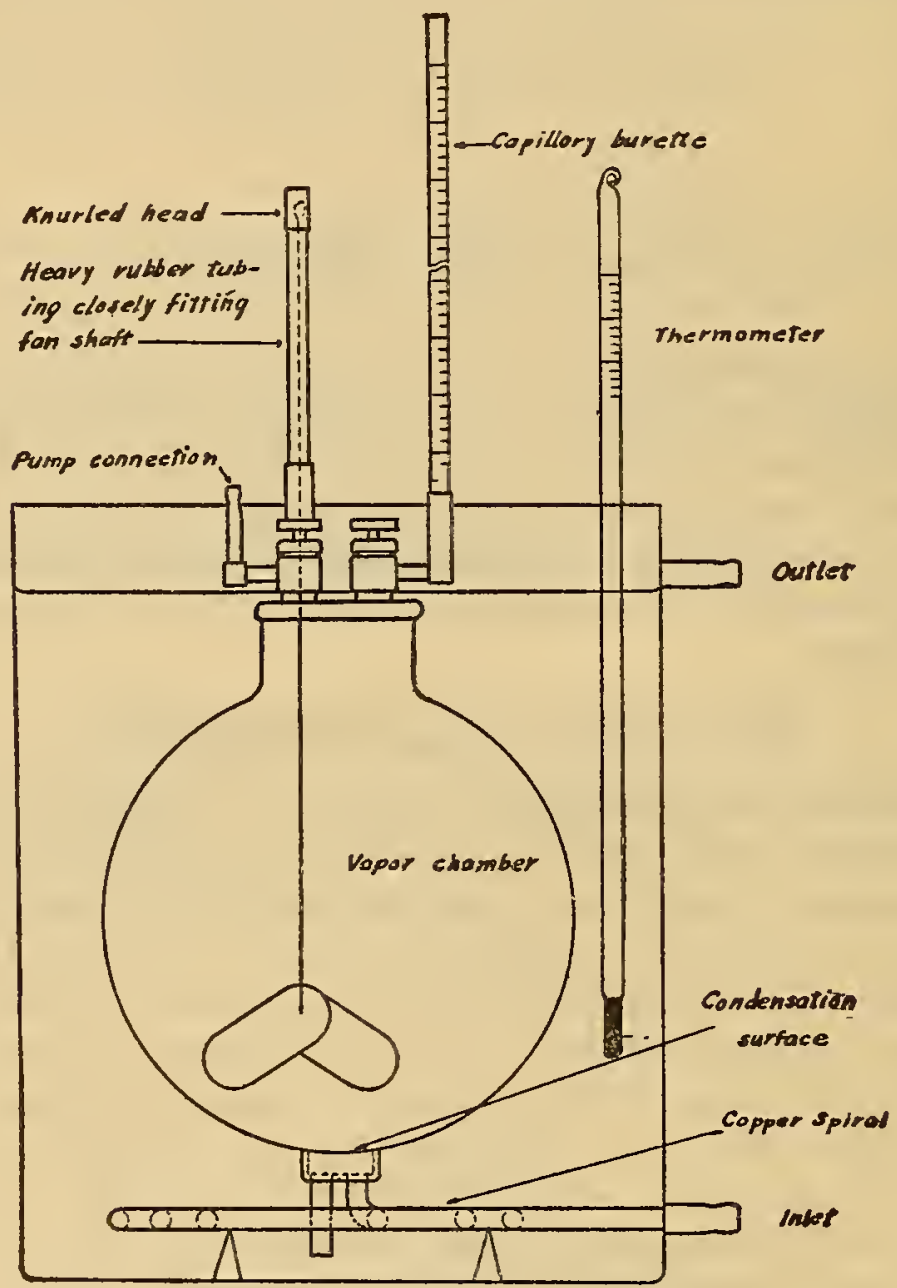

FIg. 2.-Dew-point apparatus

ated chamber, which has previously been heated a few degrees above the condensation temperature of the charge. The chamber is then cooled a few degrees below the condensation temperature by passing a stream of water into the bath. A few small drops of condensate are thus formed on the cooler spot. If necessary, the inlet water is first cooled by contact with ice. The stream is then stopped and the temperature of the bath is raised at the rate of a degree in three or four minutes. Bath and vapor are stirred continually during heating and cooling operations. The slow variation of temperature and 
thorough stirring of the mixture insure a close approach to the desired condition of equilibrium. The bath temperature at the time of disappearance of the last drop of condensate is noted and assumed to be the same as that of the vapor. The results on alcohol indicate that this assumption is sensibly correct. Two values of condensation temperature and the corresponding volumes of the liquid charge are theoretically necessary and sufficient for each fuel, but, of course, several are taken for checking purposes. The volumes employed are such as to give about the same range of partial pressures as are encountered in the engine's manifold. The condensation temperature is sharply defined; with proper precautions observations on the same charge vary by not more than half a degree centigrade.

As a second step the chamber is connected with a small closed-end mercury manometer immersed in the bath, nearly evacuated and maintained at a constant temperature high enough to completely vaporize the maximum charge employed. With this arrangement measurements are made of the pressures exerted by several measured volumes of fuel; one such measurement is theoretically sufficient. The constant ratio between the pressure and the mass of the fuel is used to compute its average molecular weight.

Measurement with a Westphal balance of the density of fuel at the temperature at which it is supplied to the chamber completes the data required.

The value of the average molecular weight $M_{\mathrm{f}}$ is computed from the data taken as the second step described above. From the equation of condition, $p v=\frac{m}{M} R T$, it follows that

$$
M_{\mathrm{r}}=\frac{\rho R T}{V} \cdot \frac{v_{\mathrm{f}}}{p_{\mathrm{p}}}
$$

where $\rho$ is the density of the liquid fuel at the temperature at which its volume is measured. The average of several values of the ratio $\frac{v_{P}}{p_{P}}$ and the measurement of $\rho, T$, and $V$ thus suffice for the computation of $M_{\mathrm{f}}$. This method is used because of its simplicity, but no great accuracy can be expected of it. However, since computed values of the dew point vary by only about $0.2^{\circ} \mathrm{C}$. per unit error in $M_{\mathrm{f}}$, the approximation so obtained is quite sufficient for the present purpose.

The value of the partial pressure, $P_{\mathrm{f}}$, for any temperature and volume of liquid fuel can now be computed from the last equation, the remaining terms of which have been evaluated for a given fuel. If logarithms of products of a convenient constant into these computed values of $P_{\mathrm{f}}$ are plotted against reciprocals of $T$, the slope of the resulting graph will, from equation (6), evidently be $K_{1}$, and $K_{2}$ 
is determined by the intercepts. Figures 3, 4, 5, and 6 are plots for the fuels tested.

The quantities $K_{1}, K_{2}$, and $M_{\mathrm{f}}$ are thus determined for each fuel, and it is only necessary to substitute them in equation (9) to have the initial condensation temperature $T$ completely defined as a function of the total pressure $P$ and the mixture ratio $R^{\prime}$ of the mixture. Figures 7, 8, 9, and 10 represent graphically the dew points computed by this relation for each of the fuels for the whole range of mixture ratios and pressures likely to be met with in practice:

\section{COMPARISON OF METHODS}

The principle of Gruse's method seems to be open to no objection. The condensation temperatures for the various mixture ratios and pressures are read directly and are sharply defined. The accuracy is limited only by that of metering and mixing the fuel and air, which operations are capable of close control. The apparatus is necessarily rather complicated.

Three difficulties are present in Wilson and Barnard's method. A practical consideration is that distillation must be continued for a long time in order to approximate closely enough a true equilibrium solution. A theoretical difficulty is that even a true equilibrium solution would be such only at the temperature at which distillation was completed, for the proportions of the constituents in the vapor phase of a solution depend on its temperature, and these proportions determine the vapor pressure. The vapor of the equilibrium solution obtained, therefore, has the same composition as the original fuel at only one temperature; at others equality of its pressure with that of the completely vaporized fuel at the dew point could only be fortuitous. The final temperature of the equilibrium solution is much higher than those at which its vapor pressure is measured; consequently the composition and pressure of its vapor at the latter temperatures might be expected to differ a good deal from those of the just completely vaporized fuel. The measured pressures are two or three times as great as our computed partial pressures for similar conditions. A relatively small error is introduced by the approximate method of determining average molecular weights of the fuels. At the time when Wilson and Barnard carried out their work there was no way of checking their results; no great accuracy was claimed for them. The apparatus employed is simple.

The method of this paper is probably somewhat less accurate than Gruse's, chiefly because errors in measuring the relatively small quantities of fuel employed are important, and because the observed initial condensation temperatures of the charges in the vapor chamber, depending as they do on the visibility of minute quantities of liquid, are not as sharply defined as in his apparatus, in which large 
quantities of fuel are used. Also, a theoretical difficulty similar to that referred to in the discussion of Wilson and Barnard's method is involved in our assumption of constancy of composition of the initial condensate at different temperatures, but the error introduced by it is negligible for the small range of temperatures involved.

A comparison of results by the three methods for various fuels at atmospheric pressure $(76 \mathrm{~cm})$ and at two mixture ratios is made in Table 1.

The condensation temperatures of alcohol secured with our apparatus agree to within less than a degree centigrade with those computed from Smithsonian Physical Tables of vapor pressures. The accuracy would be expected to decrease as the molecular weights of the substances tested increased and as the volatility decreased, as in the case of the ordinary fuel solutions. Probably a conservative estimate would be that the results obtained are correct to within about $2^{\circ} \mathrm{C}$.

TABLE 1-Comparison of dew-point determinations

\begin{tabular}{|c|c|c|c|c|c|c|c|c|c|c|c|c|}
\hline \multirow{4}{*}{ Method } & \multicolumn{12}{|c|}{ Fuels } \\
\hline & \multicolumn{2}{|c|}{$\mathbf{A}$} & \multicolumn{2}{|c|}{ B } & \multicolumn{2}{|c|}{ C } & \multicolumn{2}{|c|}{$\mathrm{X}$} & \multicolumn{2}{|c|}{$\mathbf{P}$} & \multicolumn{2}{|c|}{$\mathrm{GQT}$} \\
\hline & \multicolumn{12}{|c|}{ Mixture ratios } \\
\hline & 12 & 15 & 12 & 15 & 12 & 15 & 12 & 15 & 12 & 15 & 12 & 15 \\
\hline $\begin{array}{l}\text { Bureau of Standards } \\
\text { Gruse }\end{array}$ & $\begin{array}{l}{ }^{\circ} \mathrm{C} . \\
53.0 \\
53.7\end{array}$ & $\begin{array}{l}{ }^{\circ} \mathrm{C} . \\
46.5 \\
51.5\end{array}$ & ${ }^{\circ} \mathrm{C}$. & ${ }^{\circ} \mathrm{C}$. & ${ }^{\circ} \mathrm{C}$. & $\begin{array}{l}{ }^{\circ} \mathrm{C} . \\
66.5\end{array}$ & $\begin{array}{l}{ }^{\circ} \mathrm{C} . \\
17.0 \\
17.0\end{array}$ & $\begin{array}{l}{ }^{\circ} \mathrm{C} . \\
14.0 \\
11.0\end{array}$ & $\begin{array}{l}{ }^{\circ} \mathrm{C} . \\
69.5 \\
71.0\end{array}$ & $\begin{array}{l}{ }^{\circ} \mathrm{C} . \\
64.0 \\
64.5\end{array}$ & $\begin{array}{l}{ }^{\circ} \mathrm{C} . \\
62.5 \\
64.0\end{array}$ & $\begin{array}{r}{ }^{\circ} \mathrm{C} . \\
57.0 \\
56.0\end{array}$ \\
\hline Wilson and Barnard. & 28.5 & 24.0 & 37.0 & 32.0 & 48.5 & 43.5 & 1.0 & -4.0 & & & & \\
\hline
\end{tabular}

1 Approximate. 


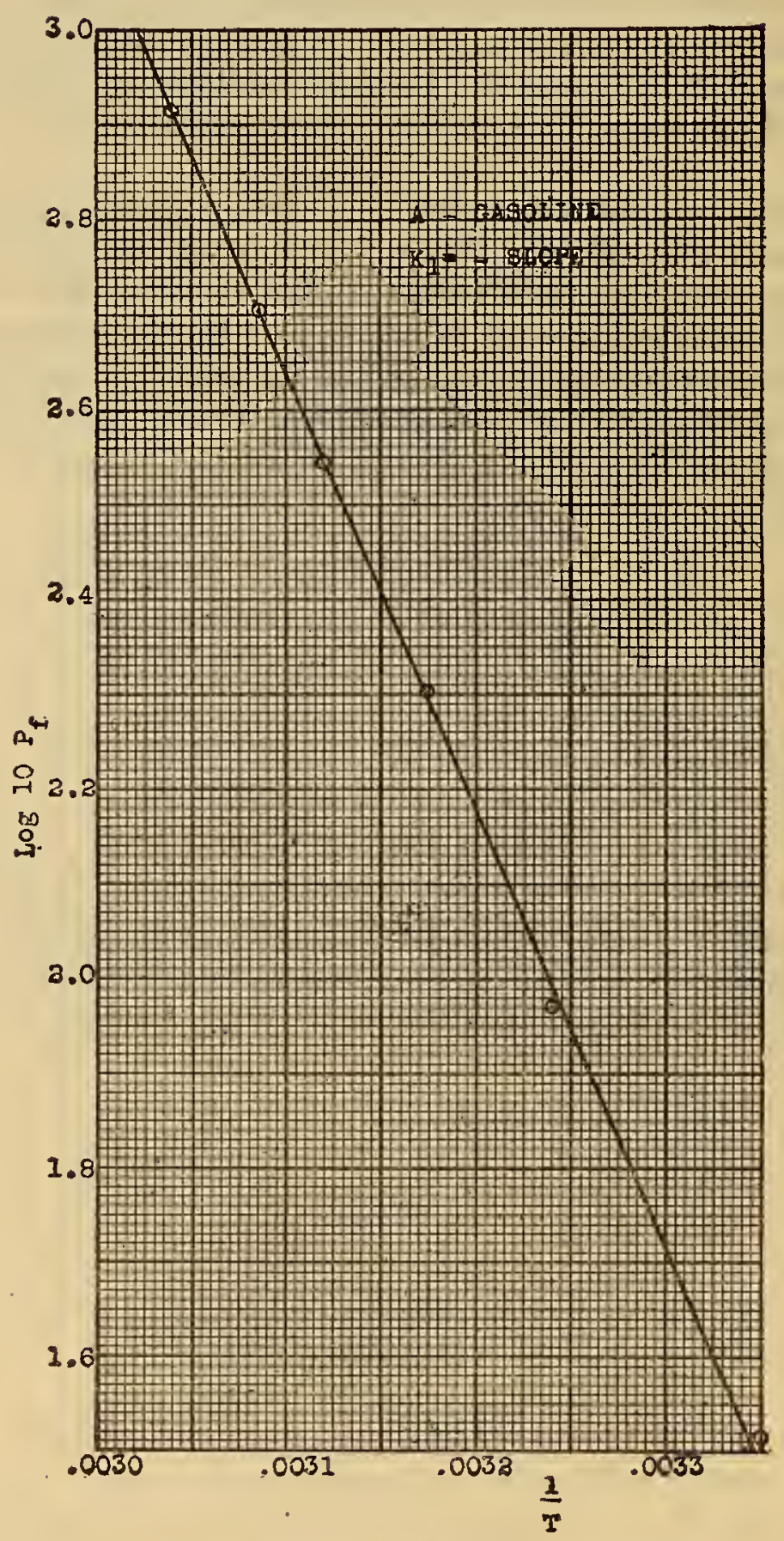

FIG. 3.-Determination of $K_{1}$ and $K_{2}, A$-gasoline

$$
K_{1}=-4550 \quad K_{2}=14.37 \quad M_{\mathrm{f}}=121
$$




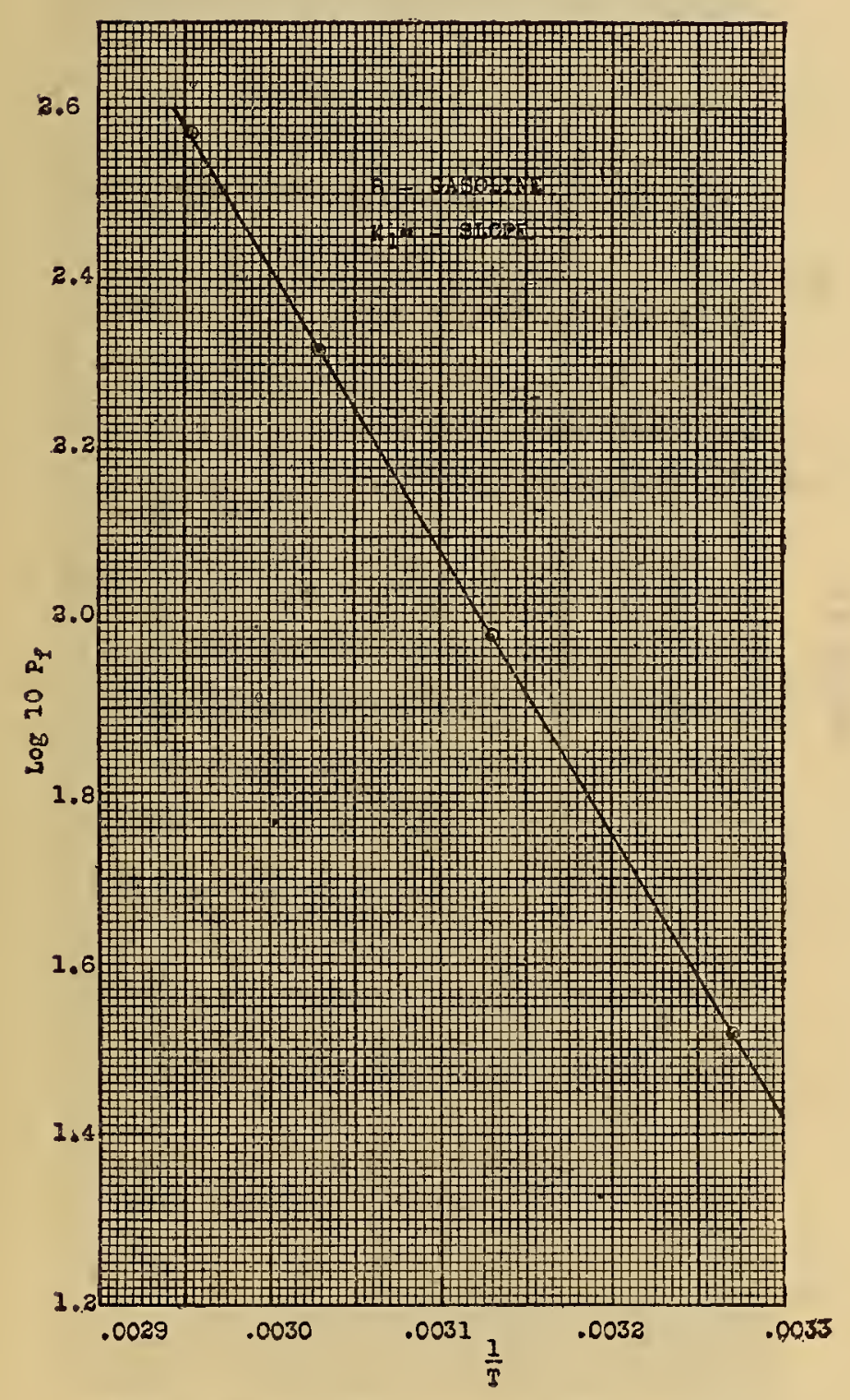

Fig. 4.-Determination of $K_{1}$ and $K_{2}, B$-gasoline $K_{1}=-3335 \quad K_{2}=10.11 \quad \Delta H_{\mathrm{f}}=124$ 


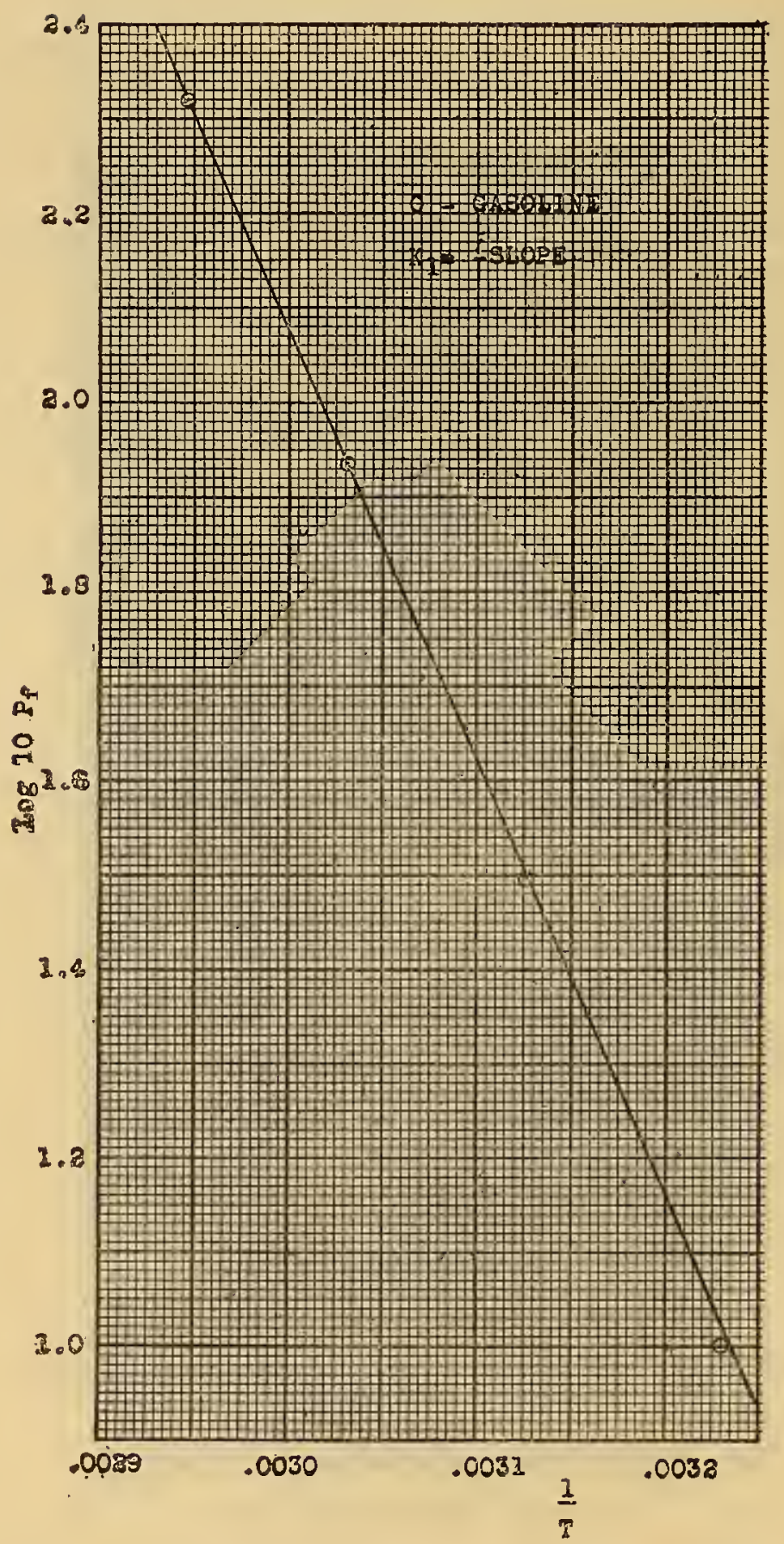

FIG. 5.-Determination of $K_{1}$ and $K_{2}, C$-gasoline $K_{1}=-4590 \quad K_{2}=13.72 \quad M_{1}=125$ 


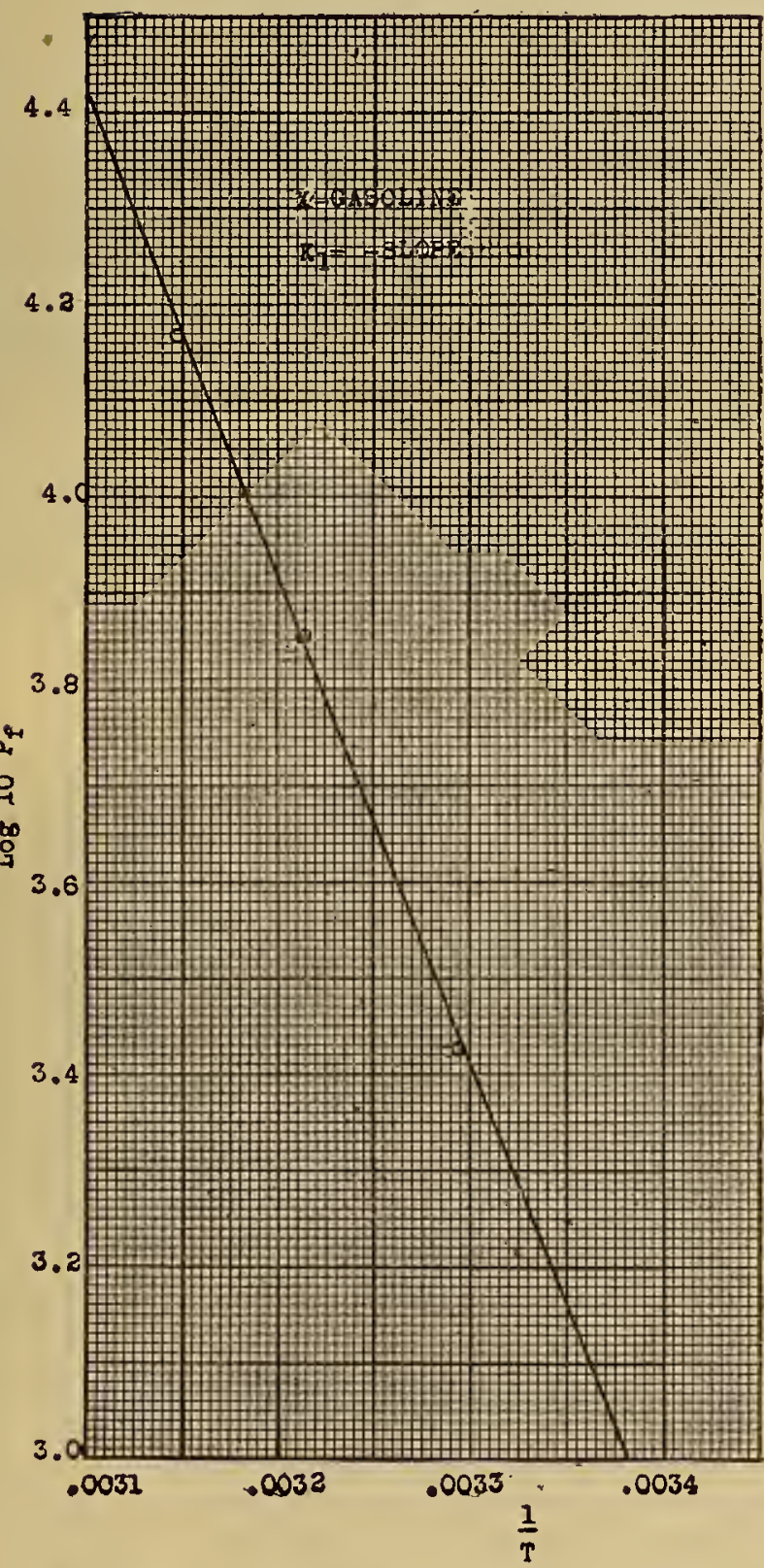

Fig. 6.-Determination of $K_{1}$ and $K_{2}, X$-gasoline $K_{1}=-5040 \quad K_{2}=17.76 \quad M_{\mathbf{f}}=120$ 


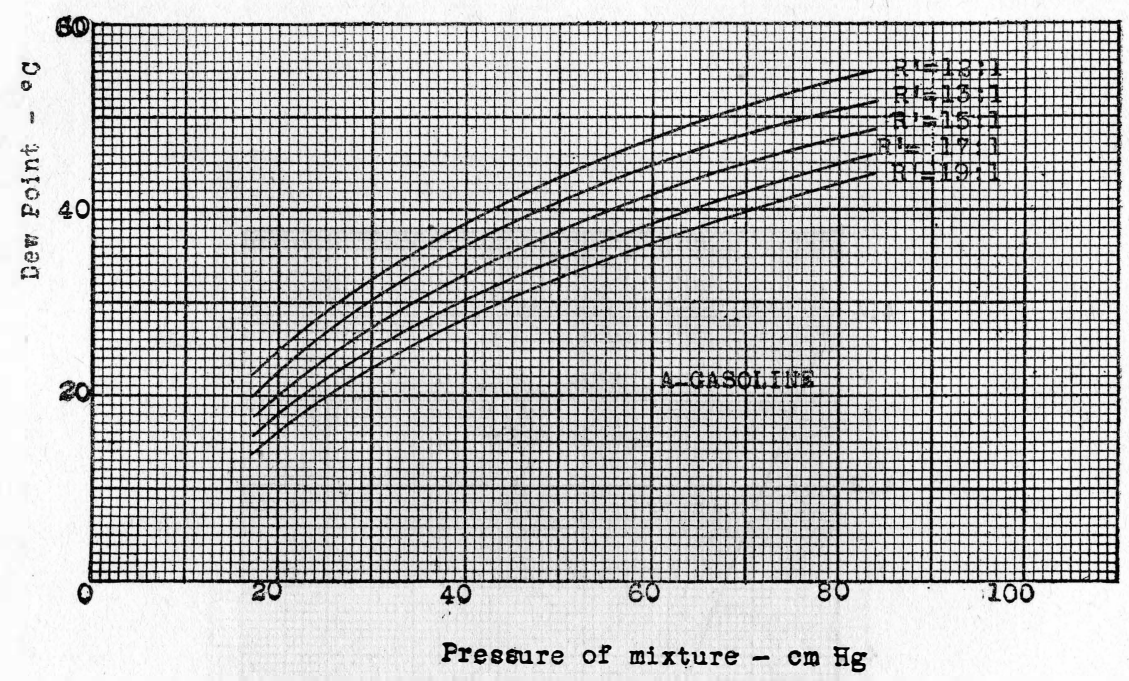

Fig. 7.-Dew-point curves for A-gasoline

Mixtureratios: 12:1, 13:1, 15:1, 17:1, 10:1

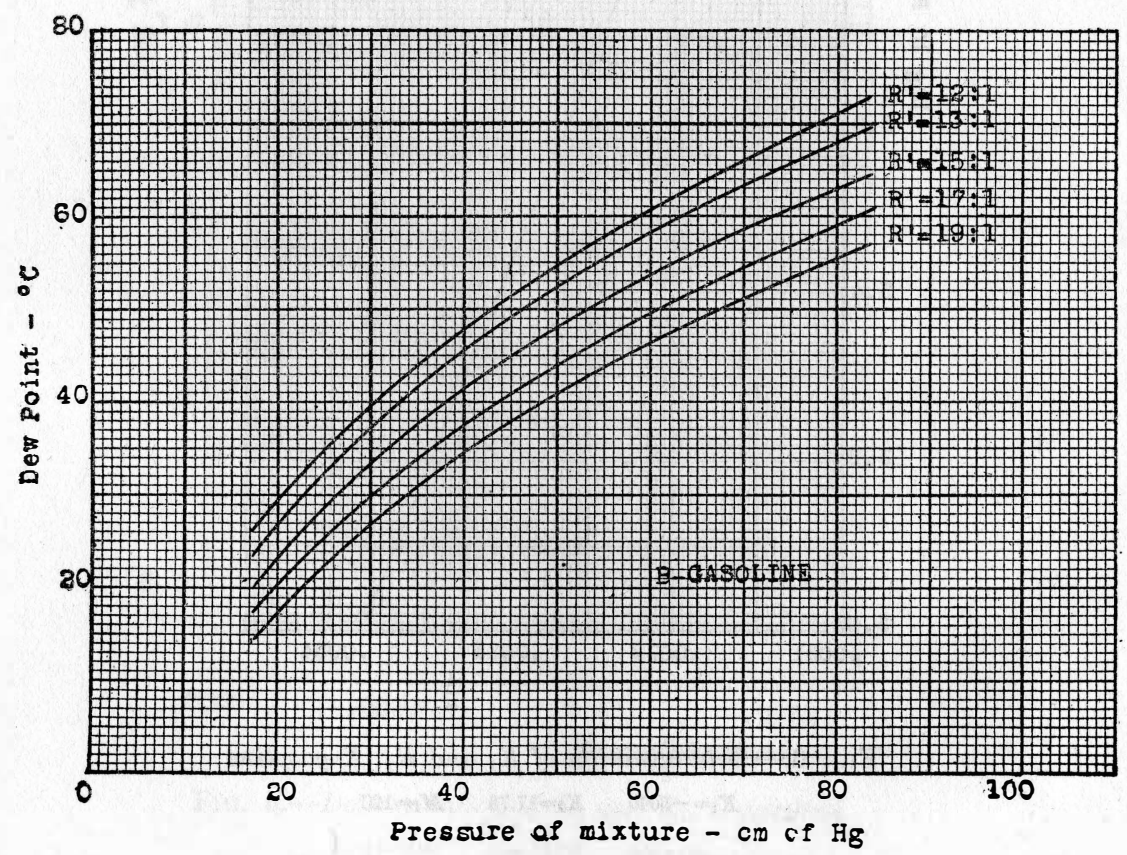

FIG. 8.-Dew-point curves for B-gasoline

Mixture ratios: 12:1, 13:1, 15:1, 17:1, 19:1 


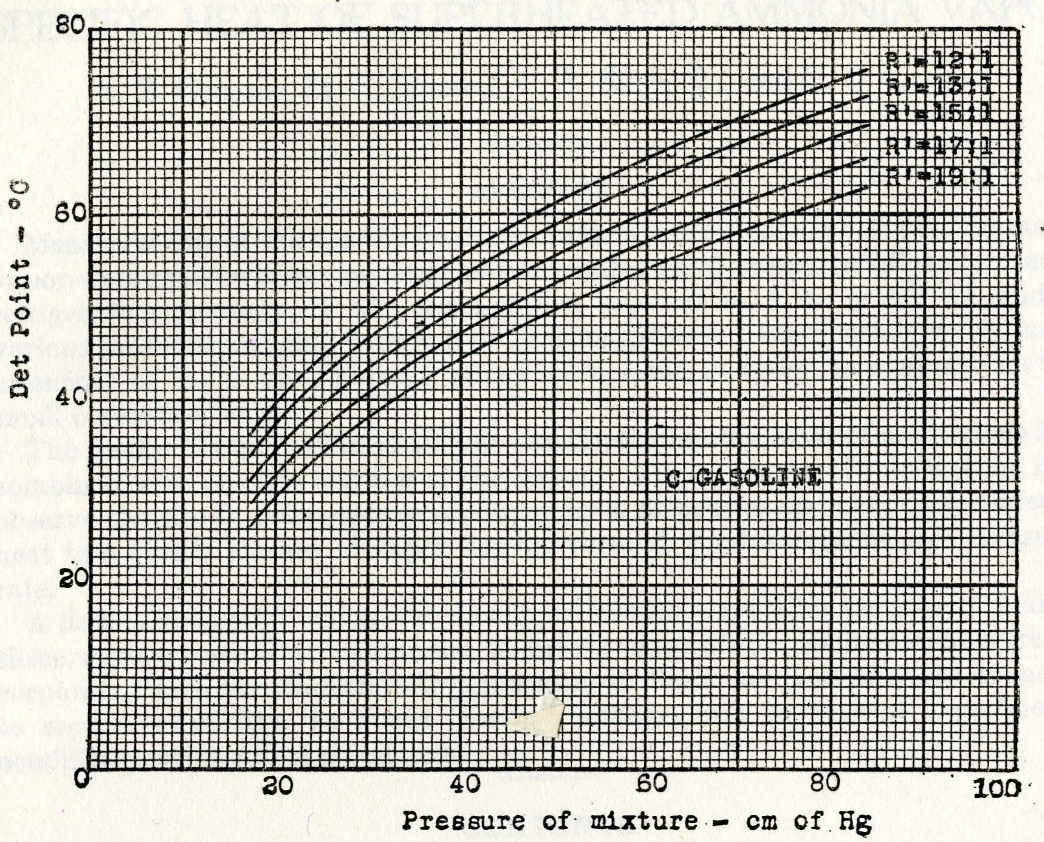

FIG. 9.-Dew-point curves for C-gasoline

Mixture ratios: 12:1, 13:1, 15:1, 17:1, 19:1

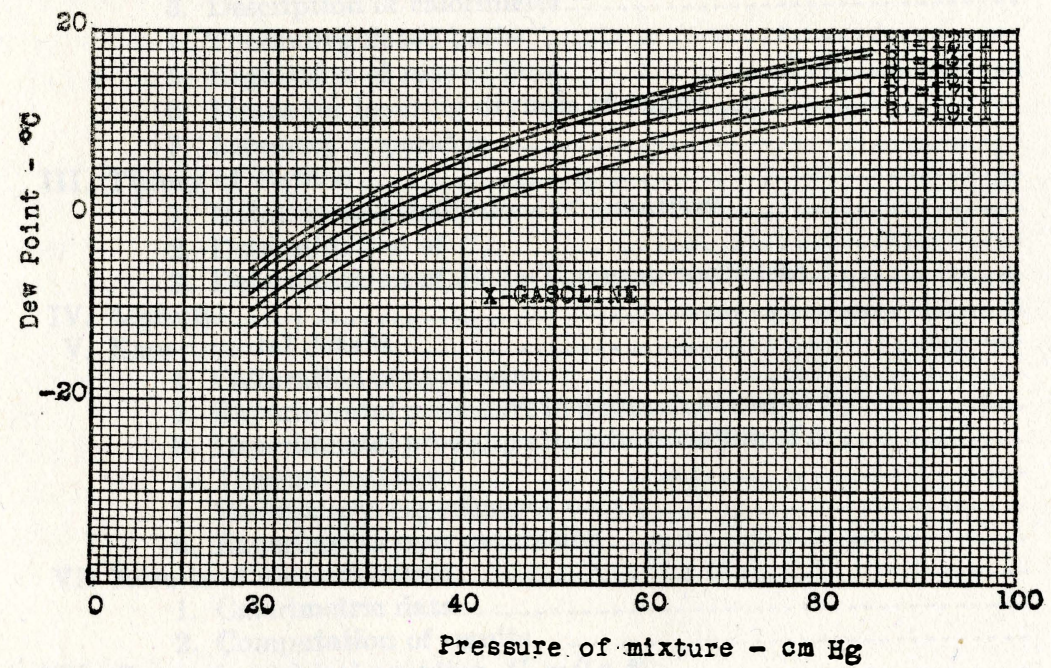

FIG. 10.-Dew-point curves for $X$-gasoline

Mixture ratios: 12:1, 13:1, 15:1, 17:1, 19:1 